\title{
Isomorphisms of finite Cayley graphs
}

\section{Cai Heng Li}

A fundamental problem in graph theory is the so-called isomorphism problem, that is, to decide whether two given graphs are isomorphic. In this thesis we investigate the isomorphism problem for finite Cayley graphs.

For a finite group $G$ and a subset $S$ of $G \backslash\{1\}$, the Cayley digraph Cay $(G, S)$ of $G$ with respect to $S$ is defined as the directed graph with vertex set $G$ and arc set $\left\{(a, b) \mid a, b \in G, b a^{-1} \in S\right\}$. If $S=S^{-1}:=\left\{s^{-1} \mid s \in S\right\}$ then Cay $(G, S)$ may be viewed as an undirected graph and is called the Cayley graph of $G$ with respect to $S$. It easily follows that Cay $(G, S)$ has valency $|S|$ and that Cay $(G, S)$ is connected if and only if $\langle S\rangle=G$.

The group $G$ acting by right multiplication (that is, $g: x \rightarrow x g$ ) is a subgroup of the automorphism group of Cay $(G, S)$ and acts regularly on vertices. If $\sigma$ is an automorphism of $G$, then $\sigma$ induces an isomorphism from Cay $(G, S)$ to Cay $\left(G, S^{\sigma}\right)$. A Cayley (di)graph Cay $(G, S)$ is called a $C I$-graph if, for any Cayley (di)graph Cay $(G, T)$ for $G$, whenever Cay $(G, S) \cong$ Cay $(G, T)$ we have $S=T^{\sigma}$ for some $\sigma \in \operatorname{Aut}(G)$. (CI stands for Cayley Invariant.)

One long-standing open problem about Cayley graphs is to determine the groups $G$ (or the types of Cayley graphs for a given group $G$ ) for which all Cayley graphs for $G$ are $C I$-graphs. The investigation of this problem was begun with a conjecture posed by Ádám [1] in 1967 that all cyclic groups had this property. This was disproved by Elspas and Turner [4] in 1970, and since then, it has received considerable attention in the literature. In this thesis we investigate the problem for general groups under various conditions.

A finite group is called an $m$-DCI-group ( $m$-CI-group) if all Cayley digraphs (graphs respectively) of $G$ of valency at most $m$ are CI-graphs. One of the main topics of the thesis is to study $m$-(D)CI-groups. It is clear from the definition that an $m$ (D)CI-group is automatically an $i$-(D)CI-group for every $i \leqslant m$. Further, a group $G$ is a 1-DCI-group if and only if all elements of $G$ of the same order are conjugate under

Received 26th February, 1997.

Thesis submitted to the University of Western Australia, July 1996. Degree approved, December 1996.

Supervisor: Professor CE Praeger.

Copyright Clearance Centre, Inc. Serial-fee code: 0004-9729/97 \$A2.00+0.00. 
Aut $(G)$, and if $G$ is a 2-CI-group then any two elements $a, b$ of $G$ of the same order are fused (namely $a^{\alpha}=b$ for some $\alpha \in$ Aut $(G)$ ) or inverse-fused (namely $a=\left(b^{-1}\right)^{\sigma}$ for some $\sigma \in \operatorname{Aut}(G))$. We call a group with the latter property an FIF-group. First we prove that a finite nonabelian simple group is an FIF-group if and only if it is $\mathrm{A}_{5}, \mathrm{~A}_{6}, \operatorname{PSL}(2,7), \operatorname{PSL}(2,8), \operatorname{PSL}(3,4), \mathrm{Sz}(8), \mathrm{M}_{11}$ or $\mathrm{M}_{23}$, and give a good description of general FIF-groups, which are dependent on the classification of finite simple groups. (These results have been written as publications $[14,15]$ ). Then we apply the description of FIF-groups to obtain explicit lists which contain $m$-DCI-groups $(m \geqslant 2)$ and $m$-CI-groups $(m \geqslant 4)$. Further we prove that a finite nonabelian simple group is a 2-CI-group if and only if it is $A_{5}$ or $\operatorname{PSL}(2,8)$, and however only $A_{5}$ is a 2-DCI-group or a 3-CI-group. Moreover we construct a 29-valency Cayley graph of $\mathrm{A}_{5}$ which is not a CI-graph so that $A_{5}$ is not a 29-CI-group. Combining these results, we prove that all (D)CI-groups are soluble, and obtain a description of (D)CI-groups which completes and improves results of Babai and Frankl in $[2,3]$ dating from 1978. (These results have been written as publications $[11,16,17]$.)

In contrast to the study of $m$-(D)CI-groups, the second topic in the thesis is to investigate finite groups which have a weaker property. A group $G$ is said to have the $m$-(D)CI property if all Cayley (di)graphs of $G$ of valency $m$ are CI-graphs. First we answer the question of whether the $m$-(D)CI property implies the $i$-(D)CI property for $1 \leqslant i<m$ by constructing, for infinitely many values of $m$, a family of Frobenius groups which have the $m$-(D)CI property but not the $i$-(D)CI property for any $i<m$. Then we prove that the 2-CI property implies the 1-CI property and, on the other hand we show that this is not so for the 2-DCI property and we give a complete classification of the finite groups with the 2-DCI property but not the 1-DCI property. Further, we make a general investigation of the structure of Sylow subgroups of groups with the $m$-(D)CI property. Finally, although it is very hard to characterize general groups with the $m$-(D)CI property, we obtain a reasonably complete classification of cyclic groups with the $m$-DCI property, and prove that every Sylow subgoup of Abelian groups with the $m$-DCI property is a homocyclic group, namely a direct product of cyclic groups of the same order. (These results have been written as publications $[\mathbf{5}, \mathbf{6}, \mathbf{7}, \mathbf{1 2}, \mathbf{1 8}]$.)

Thirdly, we study the isomorphism problem for connected Cayley graphs. A finite group $G$ is called a connected $m$ - $(D) C I$-group if all connected Cayley (di)graphs of $G$ of valency at most $m$ are CI-graphs. Let $G$ be a finite group and let $p$ be the smallest prime divisor of $|G|$. First we show that $G$ is a connected $(p-1)$-DCI-group but is not necessarily a connected $p$-DCI-group, the latter of which provides a negative answer to two conjectures posed by $\mathrm{Xu}[19]$. Then we prove that an Abelian group $G$ is a connected $p$-DCI-group but not necessarily a connected $(p+1)$-DCI-group, and we give a complete classification of Abelian $(p+1)$-DCI-groups. As a corollary, we 
obtain a complete classification of Abelian ( $p+1)$-DCI-groups, the case where $p=2$ of which gives several earlier results. Further we show that the PSL $(2, q)$ are connected 2-DCI-groups. Finally we prove that all connected symmetric cubic Cayley graphs of simple groups are CI-graphs. As a by-product, we show that, if $G$ is a nonabelian simple group and $\Gamma$ is a symmetric cubic Cayley graph for $G$, then with a few possible exceptions $G$ is normal in the full automorphism group Aut $\Gamma$. (These results have been written as publications $[\mathbf{8}, \mathbf{9}, \mathbf{1 0}, \mathbf{1 3}]$.)

Also, related to the results obtained in the thesis, a number of interesting research problems arise and remain to be solved.

\section{REFERENCES}

[1] A. Ádám, 'Research problem 2-10', J. Combin. Theory 2 (1967), 309.

[2] L. Babai and P. Frankl, 'Isomorphisms of Cayley graphs I', Colloq. Math. Soc. János Bolyai 18 (1978), 35-52.

[3] L. Babai and P. Frankl, 'Isomorphisms of Cayley graphs II', Acta Math. Hungar. 34 (1979), 177-183.

[4] B. Elspas and J. Turner, 'Graphs with circulant adjacency matrices', J. Combin. Theory 9 (1970), 297-307.

[5] C.H. Li, 'The finite groups with the 2-DCI property', Comm. Algebra 24 (1996), 1749-1757.

[6] C.H. Li, 'Finite Abelian group with the $m$-DCI property', Ars Combin. (to appear).

[7] C.H. Li, 'The cyclic group with the $m$-DCI property', European J. Combin. (to appear).

[8] C.H. Li, 'On isomophisms of connected Cayley graphs', Discrete Math. (to appear).

[9] C.H. Li, 'Isomorphisms of connected Cayley digraphs', Graphs Combin. (to appear).

[10] C.H. Li, 'On isomorphisms of connected Caylet graphs, II', (submitted).

[11] C.H. Li, 'Finite CI-groups are solvable', (submitted).

[12] C.H. Li, 'On finited groups with the Cayley isomorphism property, II', (submitted).

[13] C.H. Li, 'The automorphism group of symmetric cubic graphs', (preprint).

[14] C.H. Li and C.E. Praeger, 'The finite simple groups with at most two fusion classes of every order', Comm. Algebra 24 (1996), 3681-3704.

[15] C.H. Li and C.E. Praeger, 'On finite groups in which any two elements of the same order are fused or inverse-fused', Comm. Algebra (to appear).

[16] C.H. Li and C.E. Praeger, 'On the isomorphism problem for finite Cayley graphs of bounded valency', (preprint, 1997).

[17] C.H. Li, C.E. Praeger and M.Y. Xu, 'Isomorphisms of finite Cayley diagraphs of bounded valency', (submitted).

[18] C.H. Li, C.E. Praeger and M.Y. Xu, 'On finite groups with the Cayley isomorphism property', (submitted). 
[19] M.Y. Xu, 'Some work on vertex-transitive graphs by Chinese mathematicians', in Group theory in China (Science Press/Kluwer Academic Publishers, Beijing/New York, 1996), pp. 224-254.

Department of Mathematics

University of Western Australia

Perth WA 6907

Australia 